\title{
SCREENING OF COTTON CULTIVARS FOR DROUGHT TOLERANCE UNDER FIELD CONDITIONS
}

\author{
Volkan SEZENER ${ }^{1}$, Huseyin BASAL ${ }^{2 *}$, Ceng PEYNIRCIOGLU ${ }^{3}$, Talih GURBUZ ${ }^{4}$, Kadir KIZILKAYA ${ }^{5}$ \\ ${ }^{1}$ Nazilli Cotton Research Institute, Aydin, TURKEY \\ ${ }^{2}$ Adnan Menderes University, Faculty of Agriculture, Department of Field Crops, Aydın, TURKEY \\ ${ }^{3} \ddot{O}_{z}$ altin Seed Company, Aydın, TURKEY \\ ${ }^{4}$ Adnan Menderes University' Faculty of Agriculture, Department of Agricultural Structures and \\ Irrigation, Aydin, TURKEY \\ ${ }^{5}$ Adnan Menderes University, Faculty of Agriculture, Department of Animal Science, Aydin, TURKEY \\ *Corresponding author: hbasal@adu.edu.tr
}

Received: 31.08 .2015

\begin{abstract}
To develop cotton germplasm with improved yield under drought conditions is one of the major goals for cotton breeders. The main purpose of this study was to evaluate 96 cotton genotypes for drought tolerance by measuring yield performance under deficit (water-limited) and full irrigation conditions. The field experiment was conducted under full (FI-100) and deficit (DI-50) irrigation conditions during the two growing seasons of 2011 and 2012 at the Agricultural Research Station of Adnan Menderes University, Aydin, Turkey. The mean data on performance of 96 different cotton genotypes showed the existance of considerable genotypic variations for yield, yield components, and drought tolerance indices. Correlation and regression analysis indicated that cotton genotypes characterizied with high GMP and low DSI could be selected as a potentially droght-tolerant genotypes. It is concluded from the present studies that, based on biplot analysis, 20 genotypes were found highly susceptile to water stress, 26 genotypes were highly susceptible to water stress but produced high yield in full irrigation, and 23 genotypes were not only water stress tolerant but also give maximum seed cotton yield. However, GC 555, Nieves, DAK-66/3, MS-30/1, Nazilli M-503, Zeta 2, Eva, NIAB 999, and Delta Diomand were found highly water stress tolerant because of maximum GMP and minimum DSI values. These genotypes could be exploited as genetic resources in breeding programs aiming to improve drought tolerance in cotton.
\end{abstract}

Key words: Cotton, Gossypium hirsutum L. water stress, selection criterias

\section{INTRODUCTION}

Amongst the abiotic stresses, water stress has been considered as a threat for low crop productivity in many regions of the world (Turner, 1997; Sinclair, 2005). While demands on water resources for agricultural purposes is increasing, declining water availability, and increasing human demands are limiting its availability for agriculture. Approximately one third of the cultivated area of the world suffers from chronically inadequate supplies of water (Massacci et al., 2008). Further it is reported that future climatic changes are expected to increase risks of drought (Rizza et al., 2004).

Cotton is an excellent candidate for irrigation. With proper management, irrigation has been shown to increase lint yield by more than $350 \mathrm{~kg} \mathrm{ha}^{-1}$ in Georgia (Bednarz et al., 2002). Numerous studies have reported how cotton reproductive growth, yield and fibre quality are affected by moisture deficits. Cotton yield is dependent upon the production and retention of bolls, and both can be decreased by water stress (Guinn and Mauney, 1984). Under water stress, decrease in seed cotton yield is primarily due to the reduction in number of bolls and boll weight (Pettigrew, 2004; Wang et al., 2004; Mert, 2005; Basal et al., 2009). Water stress affects lint quality in numerous ways, especially during the fibre elongation period, which results in a decrease in fibre length and causes fibre immaturity (Ritchie et al., 2004; Mert, 2005).

Previous studies reported that there is genetic variability for drought response in cotton subjected to water deficient since cotttableon originates from areas that are often exposed to water-deficit stress (Quisenberry et al., 1981, Lacape et al., 1998; Pettigrew and Meredith, 1994). Therefore, selection for drought tolerance is a major interest of plant breeders in cotton, as well as other agricultural crop commodities. A number of different morphological (leaf, stem and root growth parameters) and physiological traits (more than 30 traits) have been suggested as important selection criteria relative to 
drought tolerance in cotton (Loka et al., 2011). However, none of the above physiological traits has so far been consistently correlated positively with drought tolerance (Loka et al., 2011). The difficulty in identification of a physiological parameter as a reliable indicator of yield in drought conditions has suggested that yield performance over a range of environments should be used as the main indicator for drought tolerance (Voltas et al., 2005).

Most of the screening studies were conducted under controlled conditions by using pot experiments. Unfortunately, pot experiments can have several serious disadvantages that make the results difficult to extrapolate to the field (Passioura, 2006). When the growing conditions are below the optimum, small variations in growing conditions will amplify differences in plant growth. Hence, pot experiments under stress conditions will increase error variance due to the pot size, the physical constraint of roots in a small container, potting mixture (media), commercial plant nutrients, shoock stress treatment

(http://www.plantstress.com/methods/PotExp.htm).

Therefore, the experiments were conducted under field conditions to quantify cotton genotypes performance for drought tolerance.

Since drought is the most significant environmental stress in global agriculture, developing germplasm with improved yield under drought conditions is a major plant breeding goal (Cattivelli et al., 2008). In order to improve such new cultivars, two basic requirements must be available. Firstly, there must be variability for water stress tolerance in the crop as a whole, and secondly, this variation must be genetically controlled. To develop cotton varieties for drought tolerance, the first step in breeding programme is to determine suitable parents. Thus, the main purpose of this study is to screen the cotton genotypes for drought tolerance by measuring yield performance under deficit (water-limited) and full irrigation conditions, and to select drought tolerant cotton genotypes that would be used as genetic resources by cotton breeders in hybridiziaton breeding programme for improving cotton productivity under drought conditions in future investigations.

\section{MATERIALS AND METHODS}

The experiments were conducted during the two growing seasons of 2011 and 2012 at the Agricultural Research Station of Adnan Menderes University, Aydin, Turkey. The longitude and latitude of the experiment site are $37^{\circ} 51^{\prime} \mathrm{N}$ and $27^{\circ} 51^{\prime} \mathrm{E}$, respectively. Climate in this region is semiarid with total annual precipitation of 657 $\mathrm{mm}$. The soil type of the experimental area was loam and sandy loam in texture. For the cotton experiment area, water content at field capacity varied from 20.3 to $27.6 \%$, and wilting point varied from 7.2 to $9.7 \%$ on dry weight basis. The dry soil bulk densities ranged from 1.42 to 1.50 $\mathrm{g} \mathrm{cm}^{-3}$ throughout the $1.2 \mathrm{~m}$ deep profile. The experiment was arranged in the augmented block design with four replications. Ninety eight (96) cotton genotypes and five check varieties (Carmen, Sahin 2000, BA 119, GSN 12, and Claudia) were used as plant material. Total 101 cotton genotypes were planted on 19 May 2011 and on 03 May 2012 respectively. Cotton plants were planted at $0.70 \mathrm{~m}$ (row width) $0.20 \mathrm{~m}$ (between plants). Each cotton genotype was planted one row with $12 \mathrm{~m}$ long and only 10 $\mathrm{m}$ length was harvested. A compound fertilizer (15\%, 15 $\%$ and $15 \%$ composite) was applied at a rate of $60 \mathrm{~kg} \mathrm{ha}^{-1}$ pure $\mathrm{N}, \mathrm{P}$ and $\mathrm{K}$ before planting. The required remaining portion of nitrogen was followed by $82 \mathrm{~kg} \mathrm{ha}^{-1}$ as ammoniumnitrate $33 \%$ before first irrigation.

The experiment included two irrigation regimes, namely full (meeting $100 \%$ of crop water needs) and deficit irrigation (meeting $50 \%$ of crop water needs). The irrigation treatments were based on replenishment of soil water depletion. The control treatment, full irrigation, (FI100) was designated to receive $100 \%$ replenishment of soil water depletion. Depletion was defined as the difference between the depth of water held in the root zone at field capacity and the depth of water actually held in the root zone at the time of irrigation. Irrigation was applied when $50 \%$ of available soil moisture was consumed in the $1.20 \mathrm{~m}$ root zone in the FI-100 treatment during the irrigation periods. The measured soil moisture level at the control (FI-100) treatment was used to initiate irrigation of cotton during the growing season. In treatments, deficit irrigation (DI-50) was applied at the rates of $50 \%$ of control treatments (FI-100) on the same day. A drip irrigation system was designated for the experiment. The average amount of applied water was about $313 \mathrm{~mm}$ for DI-50 (deficit irrigation) and $626 \mathrm{~mm}$ FI-100 (full irrigation). Soil water level was monitored by using the gravimetric method in the plots of the second replication of the various treatments. Cotton yield was determined by hand harvesting in each plot on 29 September 2011 and on 14 September 2012.

Seed cotton yield (kg ha), lint percentage (\%), boll number (per plant), and boll weight were tested for differences in genotypes. Also the drought susceptibility index (DSI) and geometric mean productivity (GMP) were calculated as follows: Drought intensity index (DI) for each cultivar was calculated as

$$
\mathrm{DI}=1-\frac{\hat{\mathrm{Y}} \mathrm{s}}{\hat{\mathrm{Y} p}}
$$

where $\hat{Y} s$ and $\hat{Y} p$ are the means of all genotypes under full irrigated and deficit irrigated conditions, respectively.

The formula proposed by Fisher and Maurer (1978) was used to calculate drought susceptibility index (DSI) for each cultivar.

$$
\mathrm{DSI}=1-\frac{\frac{Y \mathrm{~s}}{\mathrm{Yp}}}{\mathrm{DI}}
$$


Where Yp and Ys are mean yields of a given cultivar under full irrigated and deficit irrigated conditions, respectively and DI is drought intensity index.

Geometric mean productivity (GMP) was calculated by using the formula proposed by Fernandez (1992):

$$
\mathrm{GMP}=\sqrt{\mathrm{Yp} \times \mathrm{Ys}}
$$

Statistical analysis was performed using JMP 5.0.1 statistical software (SAS Institute Inc., 2002) and the means were grouped with Fisher's Least Significant Difference (LSD) test at alpha level of 0.05. Linear associations among traits of intrest were determined by estimating correlation coefficient. Also, multiple regression analysis was carried out to dermined the variables affecting the seed cotton yield under water stress conditions.

\section{RESULTS AND DISCUSSION}

Cotton genotypes had different response to the two moisture conditionsin in terms of investigated parameters (Table 1). Seed cotton yield of 96 cotton genotypes measured in full irrigation differed from each other, and ranged from 2,441 $\mathrm{kg} \mathrm{h}^{-1}$ of CABU/CS2-1-83 (No. 7) to $6,517 \mathrm{~h}^{-1}$ of NP EGE 2009 (No. 65). Under water stressed condition, seed cotton yield markedly reduced and these ranged from 1,791 $\mathrm{kg} \mathrm{h}^{-1}$ of CABU / CS2-1-83 (No. 7) to $3,993 \mathrm{~kg} \mathrm{~h}^{-1}$ of NP EGE 2,009 (No. 65) followed by DAK-66/3 (No. 53), Nazilli M-39 (No 58), and Zeta 2 (No. 77). Under deficit irrigation among the control varieties GSN 12 was in the first rank with $3,192 \mathrm{~kg} \mathrm{~h}^{-1}$ yield. While the difference in seed cotton yield between NP EGE 2009 and GSN 12 was not significant, NP EGE 2009 produced more lint yield than GSN 12 under water stress condition (data do not shown). Substantial variation in geometric mean productivity (GMP) ranging from $2,082 \mathrm{~kg} \mathrm{~h}^{-1}$ (CABU / CS2-1-83, No. 7) to $5,091 \mathrm{~kg} \mathrm{~h}^{-1}(\mathrm{NP}$ EGE 2009 (No. 65) was found among the cotton, respectively. On average, seed cotton yield of 96 cultivars was $2,977 \mathrm{~kg} \mathrm{~h}^{-1}$ in stress conditions as compared to 4076 $\mathrm{kg} \mathrm{h}^{-1}$ in non-stressed conditios. Average seed cotton yield decreased $27 \%$ in water stressed conditions. Percentage reduction of yield was different among cotton genotypes. The largest reduction in seed cotton yield due to drghout stress was in Tamcot $22(51 \%)$ (No. 51) followed by Taskent 1 (49\%) (No. 68). In contrast, Zeta 2 (No. 77) maintained its yield in both stress $\left(3677 \mathrm{~kg} \mathrm{~h}^{-1}\right)$ and nonstress $\left(3,697 \mathrm{~kg} \mathrm{~h}^{-1}\right)$, and therefore it had the lowest yield reduction $(1 \%)$ and low DSI value $(0.13)$.

Generally, the cultivars having DSI less than 1.0 presents the water stress tolerance as compared to other cultivars showing DSI values higher than 1.0. The DSI for seed cotton yield was the lowest for Zeta $2(0.13$; No. 77), followed by Nazilli 87 (0.23; No. 57), DAK 66/3 (0.26; No. 53), and NIAB 999 (0.26; No. 82). The highest DSI (1.89) was found in Taskent 1 (No. 68) followed by Tamcot 22 (No. 83), Taskent Uzbek (No. 70), and Coker 208 (No. 9). In this study, CABU/CS2-1-83 (No. 7) had DSI value (0.73) less than 1.0 , but the cultivar had the lowest yield $\left(1,791 \mathrm{~kg} \mathrm{~h}^{-1}\right)$ under stress condition. On the other hand, NP EGE 2009 (No. 65) had DSI value (1.43) greater than 1.0, however the cultivar had the highest yield under stressed condition. These results show that drought tolerance is a complex trait that can involve many different growth-related traits and genes, corresponding to different ways. Also, water stress tolerance can not be attributed to a genotype, because of its superiority for a single trait, therefore different parameters would be used to determine tolerant genotypes for water stress as suggested by Al-Hamdani and Barger (2003).

Water stress generally increased lint percentage of most genotypes. Mean lint percentage of 96 cotton genotypes was $36.3 \%$ for control (full irrigation) and 36.9 $\%$ for deficit irrigation (water stress) application. The SG 125 (No. 29) was found with highest lint percentage under both control and stress conditions. Delcerro MS-30 (No. 75) had the lowest lint percentage under both irrigation regimes. Boll number per plant declined significantly under drought condition. Data on boll number per plant revealed that cotton genotypes had different response to the two moisture conditions. The highest (14.9 boll plant $\left.{ }^{1}\right)$ and the lowest boll number ( 6.9 boll plant $\left.^{-1}\right)$ were found in Nazilli M-503 (No. 60) and Nazilli 87 (No. 57) under normal condition, respectively. Under stress conditions the highest boll number (12.1 boll plant $\left.{ }^{-1}\right)$ was of MS-30/1 (No. 54), while DPL 883 (No. 15) maintained the lowest boll number $\left(5.7\right.$ boll plant $\left.^{-1}\right)$. Average bolls per plant reduced to 8 bolls per plant in stress conditions against 10.9 bolls per plant in non-stressed conditions. With bolls per plant, boll weight is one of the important yield components in cotton. Based on boll weight data in Table 1, 96 cotton genotypes again appeared to respond differently to non-stressed and stressed conditions. The boll weight in control (full irrigation) ranged from $2.6 \mathrm{~g}$ of Nazilli 87 (No. 57) to $6.4 \mathrm{~g}$ of AZ 31 (No. 78). Boll weight under water stress markedly reduced and varied from ranged from $2.9 \mathrm{~g}$ of Tamcot 22 (No. 83) to $6.5 \mathrm{~g}$ of NIAB 999 (No. 82) (Table 2).

Correlation coefficients for seed cotton yields, lint percentages, boll numbers, and boll weights from the FI and DI environments were positive and significant (Table 3). A positive association was found between yield under full irrigation and GMP for seed cotton yield, boll number and boll weight. Yield in DI condition was positively correlated with boll number and boll weight, but was negatively correlated with drought susceptibility index. Also it was positively correlated with geometric mean for both boll number and boll weight.

Regression equation from multiple regression analysis of seed cotton yield under water stress was determined as:

$$
\text { Yield }=0.955 \text { GM - } 0.607 \text { DSI . }
$$

The results from multiple regression analysis indicated that geometric mean productivity (GMP) and drought susceptibility index (DSI) explained 65 and $35 \%$ of the variation observed in the seed cotton yield under water stress, respectively. The positive effects of geometric mean productivity (GMP) and negative effect of drought susceptibility index (DSI) on seed cotton yield indicated 
that higher value of GMP and lower value of DSI would increase seed cotton yield under stressed condition. Therefore, drought tolerant cotton genotypes could be selected based on these parameters. Previous studies suggested that the most effective approach in breeding for drought resistance in common bean (Ramirez-Vallejo and
Kelly, 1998), in soybean (Teran and Singh, 2002), and in cotton (Ullah et al., 2006) would be based first on selection for high GMP followed by selection among the high yielding individuals for low to moderate levels of the DSI.

Table 1. Mean seed cotton yield and lint percentage for 96 cotton genotypes and check varieties evaluated in full irrigation and deficit irrigation environments.

\begin{tabular}{|c|c|c|c|c|c|c|c|c|c|}
\hline \multirow[t]{2}{*}{ Num. } & \multirow[t]{2}{*}{ Genotype } & \multirow[t]{2}{*}{ Country of Origin } & \multicolumn{4}{|c|}{$\begin{array}{c}\text { Seed cotton yield } \\
\left(\mathrm{kg} \mathrm{ha}^{-1}\right)\end{array}$} & \multicolumn{3}{|c|}{$\begin{array}{c}\text { Lint Percentage } \\
(\%)\end{array}$} \\
\hline & & & ${ }^{*} \mathbf{F I}$ & DI & GMP & DSI & FI & DI & GMP \\
\hline 1 & Sealand 542 & USA & 4162 & 2724 & 3373 & 1.33 & 33.2 & 34.9 & 34.0 \\
\hline 2 & DPL 90 & USA & 3250 & 2584 & 2884 & 0.58 & 38.2 & 39.4 & 38.8 \\
\hline 3 & Acala 1517 & USA & 3271 & 2574 & 2888 & 0.61 & 34.9 & 35.9 & 35.4 \\
\hline 4 & Acala 5 & USA & 3403 & 2520 & 2921 & 0.89 & 36.5 & 35.5 & 36.0 \\
\hline 5 & Auburn M & USA & 3224 & 2209 & 2668 & 1.12 & 32.6 & 32.4 & 32.5 \\
\hline 6 & Blightmaster & USA & 4228 & 2764 & 3425 & 1.32 & 36.9 & 36.7 & 36.8 \\
\hline 7 & CABU / CS2-1-83 & USA & 2441 & 1791 & 2082 & 0.73 & 37.0 & 36.3 & 36.7 \\
\hline 8 & Carolina Queen & USA & 4341 & 3034 & 3629 & 1.16 & 38.5 & 38.3 & 38.4 \\
\hline 9 & Coker 208 & USA & 4223 & 2582 & 3315 & 1.52 & 37.4 & 37.5 & 37.4 \\
\hline 10 & Deltaopal & USA & 4146 & 3287 & 3679 & 0.77 & 38.0 & 38.7 & 38.3 \\
\hline 11 & DP-388 & USA & 3583 & 2640 & 3069 & 0.81 & 38.9 & 38.6 & 38.8 \\
\hline 12 & DPL 6 & USA & 3556 & 3012 & 3253 & 0.45 & 36.2 & 35.9 & 36.0 \\
\hline 13 & DPL 5415 & USA & 3753 & 2804 & 3236 & 0.87 & 37.9 & 37.8 & 37.9 \\
\hline 14 & DPL 882 & USA & 3449 & 2864 & 3125 & 0.53 & 36.6 & 36.0 & 36.3 \\
\hline 15 & DPL 883 & USA & 3925 & 2939 & 3390 & 0.86 & 35.0 & 35.1 & 35.1 \\
\hline 16 & DPL 20 & USA & 3653 & 2774 & 3174 & 0.84 & 38.9 & 37.6 & 38.2 \\
\hline 17 & DPL 886 & USA & 4744 & 2967 & 3763 & 1.42 & 37.4 & 36.3 & 36.8 \\
\hline 18 & DPL C-37 Prima & USA & 3818 & 2588 & 3146 & 1.12 & 36.4 & 34.6 & 35.5 \\
\hline 19 & DPL SR-383 & USA & 3524 & 2447 & 2936 & 1.19 & 35.9 & 33.2 & 34.5 \\
\hline 20 & GC 262 & USA & 4241 & 2865 & 3489 & 1.04 & 37.6 & 37.3 & 37.4 \\
\hline 21 & GC 555 & USA & 4634 & 3492 & 4016 & 0.79 & 36.6 & 36.4 & 36.5 \\
\hline 22 & GSA-78 & USA & 4100 & 2769 & 3373 & 1.19 & 36.7 & 35.0 & 35.8 \\
\hline 23 & Lankart 57 & USA & 4232 & 3131 & 3635 & 1.03 & 38.7 & 36.6 & 37.6 \\
\hline 24 & McNair 220 & USA & 4075 & 2981 & 3481 & 0.99 & 37.0 & 39.0 & 38.0 \\
\hline 25 & Paymaster 404 & USA & 4012 & 2818 & 3362 & 1.07 & 36.5 & 37.1 & 36.8 \\
\hline 26 & $\operatorname{Rex} 1$ & USA & 4178 & 3034 & 3561 & 1.03 & 34.2 & 36.5 & 35.3 \\
\hline 27 & S.J.V. VisaliaElmer & USA & 3603 & 2709 & 3126 & 0.87 & 36.0 & 38.0 & 37.0 \\
\hline 28 & SG 1001 & USA & 4373 & 2773 & 3481 & 1.36 & 37.9 & 38.5 & 38.2 \\
\hline 29 & SG 125 & USA & 4856 & 3011 & 3822 & 1.36 & 40.3 & 42.5 & 41.4 \\
\hline 30 & Stoneville 213 & USA & 3792 & 2867 & 3299 & 0.94 & 35.6 & 39.0 & 37.2 \\
\hline 31 & Stoneville 453 & USA & 4042 & 3209 & 3604 & 0.75 & 33.6 & 35.0 & 34.3 \\
\hline 32 & Stoneville 8751 & USA & 4254 & 2731 & 3407 & 1.25 & 37.0 & 38.3 & 37.7 \\
\hline 33 & Tamcot CABCS & USA & 3621 & 2781 & 3176 & 0.92 & 36.1 & 36.8 & 36.4 \\
\hline 34 & TKY 9309 & USA & 4705 & 2983 & 3745 & 1.26 & 35.8 & 36.4 & 36.1 \\
\hline 35 & TKY 9409 & USA & 3681 & 2390 & 2965 & 1.33 & 38.5 & 40.6 & 39.6 \\
\hline 36 & TKY 9304 & USA & 2846 & 2395 & 2615 & 0.61 & 37.1 & 35.6 & 36.3 \\
\hline 37 & Togo & S. Africa & 3663 & 3049 & 3346 & 0.63 & 34.6 & 35.1 & 34.9 \\
\hline 38 & Samon & Albanian & 3549 & 2661 & 3075 & 0.89 & 34.2 & 34.4 & 34.3 \\
\hline 39 & N-727 CC & Australia & 4422 & 2792 & 3512 & 1.38 & 38.9 & 40.0 & 39.4 \\
\hline 40 & Nieves & Australia & 4022 & 3442 & 3725 & 0.56 & 35.8 & 38.2 & 37.0 \\
\hline 41 & Semu SS/G & Australia & 3953 & 2447 & 3109 & 1.33 & 32.9 & 34.1 & 33.5 \\
\hline 42 & Sicala 3/2 & Australia & 3791 & 3332 & 3559 & 0.53 & 33.5 & 34.6 & 34.1 \\
\hline 43 & Sicala 33 & Australia & 4064 & 3043 & 3518 & 1.03 & 35.4 & 36.5 & 35.9 \\
\hline 44 & Sahel 1 & Iran & 3480 & 2693 & 3063 & 0.94 & 37.0 & 38.0 & 37.5 \\
\hline 45 & Veramine & Iran & 4290 & 3190 & 3701 & 1.02 & 33.7 & 34.9 & 34.3 \\
\hline 46 & Corona & Spain & 3128 & 2681 & 2901 & 0.53 & 37.5 & 38.6 & 38.1 \\
\hline 47 & Lachata & Spain & 3916 & 3252 & 3572 & 0.71 & 36.0 & 36.9 & 36.5 \\
\hline
\end{tabular}


Table 1. Continious

\begin{tabular}{|c|c|c|c|c|c|c|c|c|c|}
\hline \multirow[t]{2}{*}{ Num. } & \multirow[t]{2}{*}{ Genotype } & \multirow[t]{2}{*}{ Country of Origin } & \multicolumn{4}{|c|}{$\begin{array}{c}\text { Seed cotton yield } \\
\left(\mathrm{kg} \mathrm{ha}^{-1}\right)\end{array}$} & \multicolumn{3}{|c|}{$\begin{array}{c}\text { Lint Percentage } \\
(\%)\end{array}$} \\
\hline & & & ${ }^{*} \mathbf{F I}$ & DI & GMP & DSI & FI & DI & GMP \\
\hline 48 & Nata & Spain & 4020 & 2778 & 3342 & 1.26 & 35.4 & 37.2 & 36.3 \\
\hline 49 & Vulcano & Spain & 3425 & 3041 & 3233 & 0.43 & 34.8 & 37.8 & 36.3 \\
\hline 50 & Erşan 92 & Turkey & 3649 & 3313 & 3492 & 0.44 & 37.4 & 37.8 & 37.6 \\
\hline 51 & Sayar 314 & Turkey & 4016 & 3469 & 3745 & 0.61 & 36.7 & 37.8 & 37.3 \\
\hline 52 & Ayhan 107 & Turkey & 3345 & 2876 & 3113 & 0.62 & 37.0 & 36.4 & 36.7 \\
\hline 53 & DAK-66/3 & Turkey & 3986 & 3825 & 3923 & 0.26 & 37.4 & 37.9 & 37.6 \\
\hline 54 & MS-30/1 & Turkey & 4061 & 3688 & 3885 & 0.47 & 35.3 & 35.8 & 35.5 \\
\hline 55 & Nazilli 143 & Turkey & 4988 & 3592 & 4234 & 1.05 & 37.6 & 36.6 & 37.1 \\
\hline 56 & Nazilli 84-S & Turkey & 3911 & 2901 & 3371 & 0.94 & 39.8 & 40.7 & 40.2 \\
\hline 57 & Nazilli 87 & Turkey & 3546 & 3456 & 3519 & 0.23 & 35.2 & 33.9 & 34.5 \\
\hline 58 & Nazilli M-39 & Turkey & 5332 & 3700 & 4440 & 1.07 & 34.1 & 36.1 & 35.1 \\
\hline 59 & Nazilli M-503(93-7) & Turkey & 4420 & 3496 & 3937 & 0.9 & 34.0 & 33.1 & 33.6 \\
\hline 60 & Nazilli M-503 & Turkey & 4817 & 3351 & 4016 & 1.23 & 32.7 & 32.2 & 32.4 \\
\hline 61 & NGF-63 & Turkey & 4157 & 3231 & 3670 & 0.83 & 37.9 & 37.7 & 37.7 \\
\hline 62 & Barut 2005 & Turkey & 4911 & 3683 & 4256 & 0.98 & 37.2 & 37.6 & 37.4 \\
\hline 63 & Menderes 2005 & Turkey & 4048 & 2805 & 3367 & 1.19 & 36.8 & 36.3 & 36.6 \\
\hline 64 & NAPA 122 & Turkey & 3975 & 3129 & 3533 & 0.89 & 37.9 & 38.9 & 38.4 \\
\hline 65 & NP Ege 2009 & Turkey & 6517 & 3993 & 5091 & 1.43 & 36.3 & 38.5 & 37.3 \\
\hline 66 & NP Özbek 100 & Turkey & 4921 & 3599 & 4210 & 0.96 & 37.3 & 37.5 & 37.4 \\
\hline 67 & SamarkantUzbek & Uzbekistan & 4044 & 2709 & 3305 & 1.39 & 34.7 & 34.4 & 34.5 \\
\hline 68 & Taşkent 1 & Uzbekistan & 4135 & 2117 & 2933 & 1.89 & 36.0 & 36.4 & 36.2 \\
\hline 69 & Taşkent-6 & Uzbekistan & 3795 & 2881 & 3310 & 0.8 & 35.5 & 36.2 & 35.8 \\
\hline 70 & Taşkent Uzbek & Uzbekistan & 4493 & 2594 & 3398 & 1.72 & 31.3 & 33.5 & 32.3 \\
\hline 71 & $152 \mathrm{~F}$ & Uzbekistan & 3483 & 2727 & 3088 & 0.83 & 34.0 & 34.4 & 34.2 \\
\hline 72 & Aleppo-1 & Syria & 4594 & 2927 & 3659 & 1.4 & 30.7 & 32.8 & 31.7 \\
\hline 73 & S-9 & Syria & 4158 & 2847 & 3433 & 1.12 & 34.1 & 35.3 & 34.7 \\
\hline 74 & Delcerro & USA & 2675 & 2611 & 2641 & 0.33 & 34.8 & 35.1 & 34.9 \\
\hline 75 & Delcerro MS-30 & USA & 3061 & 2692 & 2867 & 0.41 & 30.7 & 31.8 & 31.3 \\
\hline 76 & Sindos 80 & Greece & 3580 & 2825 & 3175 & 0.74 & 36.4 & 35.8 & 36.1 \\
\hline 77 & Zeta 2 & Greece & 3697 & 3677 & 3686 & 0.13 & 35.9 & 36.0 & 35.9 \\
\hline 78 & AZ 31 & Israil & 5112 & 3387 & 4152 & 1.02 & 35.4 & 34.7 & 35.0 \\
\hline 79 & Eva & Greece & 4441 & 3467 & 3918 & 0.64 & 35.7 & 36.5 & 36.0 \\
\hline 80 & GW Teks & USA & 4508 & 3106 & 3734 & 1.1 & 38.0 & 37.3 & 37.7 \\
\hline 81 & NIAB 111 & Pakistan & 4037 & 3156 & 3564 & 0.72 & 37.6 & 37.9 & 37.7 \\
\hline 82 & NIAB 999 & Pakistan & 3947 & 3468 & 3696 & 0.26 & 37.4 & 37.7 & 37.5 \\
\hline 83 & Tamcot 22 & USA & 4670 & 2292 & 3256 & 1.73 & 35.6 & 35.8 & 35.7 \\
\hline 84 & Tamcot Sphinx & USA & 4214 & 2986 & 3540 & 0.88 & 34.8 & 35.0 & 34.9 \\
\hline 85 & SJ- U 86 & USA & 5549 & 3135 & 4159 & 1.47 & 35.9 & 37.7 & 36.8 \\
\hline 86 & Candia & Australia & 4427 & 3039 & 3660 & 0.76 & 40.2 & 41.5 & 40.8 \\
\hline 87 & Celia & Australia & 3931 & 2616 & 3198 & 1.18 & 36.4 & 38.9 & 37.6 \\
\hline 88 & Elsa & Australia & 5062 & 3436 & 4163 & 1.05 & 38.9 & 40.6 & 39.7 \\
\hline 89 & Delta Diomand & Spain & 4913 & 3349 & 4048 & 0.87 & 37.3 & 38.3 & 37.8 \\
\hline 90 & Gloria & Australia & 4775 & 3154 & 3872 & 1.09 & 39.7 & 38.9 & 39.3 \\
\hline 91 & Julia & Australia & 4230 & 3093 & 3610 & 0.91 & 38.9 & 39.8 & 39.3 \\
\hline 92 & Flora & Australia & 4277 & 2824 & 3467 & 1.14 & 37.0 & 36.9 & 36.9 \\
\hline 93 & PG 2018 & Turkey & 4757 & 3347 & 3983 & 0.94 & 38.6 & 40.9 & 39.7 \\
\hline 94 & BA 308 & Turkey & 4349 & 3009 & 3610 & 1.04 & 36.6 & 37.3 & 36.9 \\
\hline 95 & BA 525 & Turkey & 4583 & 3131 & 3780 & 1.04 & 39.0 & 40.4 & 39.6 \\
\hline \multirow[t]{10}{*}{96} & Lider & Turkey & 3583 & 2637 & 3067 & 0.89 & 39.3 & 41.6 & 40.4 \\
\hline & Mean & & 4076 & 2977 & 3474 & 0.95 & 36.3 & 36.9 & 36.6 \\
\hline & \multicolumn{9}{|l|}{ Check Varieties } \\
\hline & Carmen & & 3699 & 2932 & 3288 & 0.79 & 38.0 & 38.0 & 38.0 \\
\hline & Şahin 2000 & & 4536 & 2840 & 3582 & 1.44 & 34.6 & 33.0 & 33.8 \\
\hline & BA 119 & & 4102 & 3045 & 3516 & 0.83 & 38.9 & 41.3 & 40.1 \\
\hline & GSN 12 & & 4261 & 3192 & 3685 & 0.84 & 37.7 & 39.7 & 38.7 \\
\hline & Claudia & & 3765 & 2732 & 3205 & 0.96 & 40.4 & 40.5 & 40.4 \\
\hline & Mean & & 4073 & 2948 & 3455 & 0.97 & 37.9 & 38.5 & 38.2 \\
\hline & $\operatorname{LSD}(0.05)$ & & 1230 & 902 & 972 & 1.12 & 2.4 & 2.8 & 1.4 \\
\hline
\end{tabular}

"FI: Full irrigation, DI: Deficit irrigation, GMP: Geometric mean productivity, DSI: drought susceptibility index. 
Table 2. Mean boll number and boll weight for 96 cottongenotypes and check varieties evaluated in full irrigation and deficit irrigation environments.

\begin{tabular}{|c|c|c|c|c|c|c|c|c|}
\hline \multirow[t]{2}{*}{ Num. } & \multirow{2}{*}{ Genotype } & \multirow{2}{*}{ Country of Origin } & \multicolumn{3}{|c|}{$\begin{array}{l}\text { Boll number } \\
\left(\text { Boll plant }^{-1}\right)\end{array}$} & \multicolumn{3}{|c|}{$\begin{array}{l}\text { Boll weight } \\
\text { (g) }\end{array}$} \\
\hline & & & ${ }^{*} \mathbf{F I}$ & DI & GMP & FI & DI & GMP \\
\hline 1 & Sealand 542 & USA & 9.8 & 7.8 & 8.7 & 6.0 & 5.1 & 5.5 \\
\hline 2 & DPL 90 & USA & 10.5 & 7.5 & 8.9 & 4.1 & 5.0 & 4.6 \\
\hline 3 & Acala 1517 & USA & 11.4 & 7.9 & 9.5 & 4.3 & 4.4 & 4.4 \\
\hline 4 & Acala 5 & USA & 9.6 & 5.9 & 7.6 & 5.8 & 6.0 & 5.9 \\
\hline 5 & Auburn M & USA & 11.5 & 8.0 & 9.6 & 4.1 & 4.5 & 4.3 \\
\hline 6 & Blightmaster & USA & 10.2 & 6.9 & 8.4 & 5.2 & 4.5 & 4.8 \\
\hline 7 & CABU / CS2-1-83 & USA & 12.4 & 6.2 & 8.9 & 3.1 & 4.3 & 3.6 \\
\hline 8 & Carolina Queen & USA & 12.5 & 6.2 & 9.0 & 4.9 & 6.0 & 5.4 \\
\hline 9 & Coker 208 & USA & 9.4 & 7.6 & 8.4 & 6.1 & 4.7 & 5.3 \\
\hline 10 & Deltaopal & USA & 11.4 & 9.1 & 10.2 & 4.7 & 4.6 & 4.6 \\
\hline 11 & DP-388 & USA & 11.3 & 6.9 & 8.9 & 4.0 & 4.9 & 4.5 \\
\hline 12 & DPL 6 & USA & 10.8 & 6.0 & 8.2 & 5.0 & 5.6 & 5.3 \\
\hline 13 & DPL 5415 & USA & 11.0 & 7.8 & 9.3 & 4.9 & 4.8 & 4.8 \\
\hline 14 & DPL 882 & USA & 9.8 & 5.9 & 7.7 & 5.0 & 6.2 & 5.6 \\
\hline 15 & DPL 883 & USA & 9.6 & 5.7 & 7.5 & 5.5 & 5.9 & 5.7 \\
\hline 16 & DPL 20 & USA & 8.3 & 6.5 & 7.3 & 4.8 & 5.5 & 5.1 \\
\hline 17 & DPL 886 & USA & 13.2 & 8.6 & 10.7 & 4.6 & 5.0 & 4.8 \\
\hline 18 & DPL C-37 Prima & USA & 9.7 & 6.5 & 7.9 & 4.7 & 5.5 & 5.1 \\
\hline 19 & DPL SR-383 & USA & 10.8 & 7.0 & 8.7 & 4.7 & 4.9 & 4.8 \\
\hline 20 & GC 262 & USA & 10.8 & 7.1 & 8.8 & 5.4 & 5.5 & 5.5 \\
\hline 21 & GC 555 & USA & 11.8 & 7.5 & 9.5 & 5.6 & 5.8 & 5.7 \\
\hline 22 & GSA-78 & USA & 10.0 & 6.8 & 8.3 & 6.0 & 5.7 & 5.9 \\
\hline 23 & Lankart 57 & USA & 11.4 & 6.9 & 9.0 & 5.7 & 5.5 & 5.6 \\
\hline 24 & McNair 220 & USA & 11.2 & 7.9 & 9.4 & 4.9 & 5.0 & 5.0 \\
\hline 25 & Paymaster 404 & USA & 11.5 & 6.6 & 8.8 & 4.1 & 5.3 & 4.6 \\
\hline 26 & $\operatorname{Rex} 1$ & USA & 8.2 & 7.2 & 7.7 & 5.9 & 5.9 & 5.9 \\
\hline 27 & S.J.V. VisaliaElmer & USA & 11.9 & 7.7 & 9.6 & 4.2 & 5.0 & 4.6 \\
\hline 28 & SG 1001 & USA & 10.2 & 7.5 & 8.7 & 5.0 & 5.0 & 5.0 \\
\hline 29 & SG 125 & USA & 13.7 & 8.4 & 10.7 & 4.0 & 5.7 & 4.8 \\
\hline 30 & Stoneville 213 & USA & 10.5 & 6.7 & 8.4 & 4.6 & 4.8 & 4.7 \\
\hline 31 & Stoneville 453 & USA & 13.8 & 10.1 & 11.8 & 4.0 & 4.8 & 4.4 \\
\hline 32 & Stoneville 8751 & USA & 8.7 & 7.0 & 7.8 & 6.2 & 4.8 & 5.4 \\
\hline 33 & Tamcot CABCS & USA & 10.0 & 7.6 & 8.7 & 4.7 & 5.0 & 4.8 \\
\hline 34 & TKY 9309 & USA & 11.7 & 6.6 & 8.8 & 4.8 & 5.3 & 5.0 \\
\hline 35 & TKY 9409 & USA & 9.8 & 5.8 & 7.5 & 4.6 & 5.2 & 4.9 \\
\hline 36 & TKY 9304 & USA & 8.2 & 6.8 & 7.4 & 4.0 & 4.2 & 4.1 \\
\hline 37 & Togo & S. Africa & 10.9 & 8.1 & 9.4 & 4.0 & 4.3 & 4.1 \\
\hline 38 & Samon & Albanian & 9.4 & 7.3 & 8.3 & 4.7 & 5.4 & 5.0 \\
\hline 39 & N-727 CC & Australia & 12.9 & 8.9 & 10.7 & 3.2 & 4.2 & 3.7 \\
\hline 40 & Nieves & Australia & 13.8 & 7.9 & 10.4 & 4.5 & 6.1 & 5.3 \\
\hline 41 & Semu SS/G & Australia & 10.6 & 9.2 & 9.8 & 5.7 & 3.9 & 4.6 \\
\hline 42 & Sicala 3/2 & Australia & 13.4 & 9.8 & 11.4 & 3.2 & 4.6 & 3.9 \\
\hline 43 & Sicala 33 & Australia & 13.1 & 8.1 & 10.3 & 3.7 & 4.7 & 4.2 \\
\hline 44 & Sahel 1 & Iran & 8.4 & 7.9 & 8.1 & 4.1 & 4.8 & 4.5 \\
\hline 45 & Veramine & Iran & 11.7 & 7.4 & 9.3 & 4.2 & 4.9 & 4.5 \\
\hline 46 & Corona & Spain & 12.9 & 6.8 & 9.4 & 3.0 & 4.5 & 3.7 \\
\hline 47 & Lachata & Spain & 10.4 & 8.9 & 9.6 & 4.4 & 5.1 & 4.7 \\
\hline 48 & Nata & Spain & 13.4 & 9.6 & 11.4 & 3.7 & 4.5 & 4.1 \\
\hline 49 & Vulcano & Spain & 10.0 & 7.1 & 8.4 & 4.2 & 5.2 & 4.7 \\
\hline 50 & Erşan 92 & Turkey & 10.2 & 10.2 & 10.3 & 4.5 & 4.7 & 4.6 \\
\hline 51 & Sayar 314 & Turkey & 9.9 & 10.1 & 10.1 & 4.9 & 4.8 & 4.9 \\
\hline 52 & Ayhan 107 & Turkey & 8.1 & 10.7 & 9.5 & 5.4 & 3.2 & 4.2 \\
\hline 53 & DAK-66/3 & Turkey & 11.0 & 10.4 & 10.8 & 4.1 & 5.1 & 4.6 \\
\hline 54 & MS-30/1 & Turkey & 14.3 & 12.1 & 13.2 & 4.4 & 3.4 & 3.9 \\
\hline 55 & Nazilli 143 & Turkey & 10.3 & 9.1 & 9.7 & 5.6 & 4.6 & 5.1 \\
\hline
\end{tabular}


Table 2. Continious

\begin{tabular}{|c|c|c|c|c|c|c|c|c|}
\hline \multirow[t]{2}{*}{ Num. } & \multirow[t]{2}{*}{ Genotype } & \multirow[t]{2}{*}{ Country of Origin } & \multicolumn{3}{|c|}{$\begin{array}{l}\text { Boll number } \\
\left.\text { Boll plant }^{-1}\right)\end{array}$} & \multicolumn{3}{|c|}{$\begin{array}{c}\text { Boll weight } \\
\text { (g) }\end{array}$} \\
\hline & & & ${ }^{*} \mathbf{F I}$ & DI & GMP & FI & DI & GMP \\
\hline 56 & Nazilli 84-S & Turkey & 11.3 & 9.3 & 10.3 & 4.1 & 3.9 & 4.0 \\
\hline 57 & Nazilli 87 & Turkey & 6.9 & 8.2 & 15.3 & 2.6 & 6.0 & 4.0 \\
\hline 58 & Nazilli M-39 & Turkey & 11.6 & 9.2 & 10.3 & 5.4 & 4.9 & 5.2 \\
\hline 59 & Nazilli M-503(93-7) & Turkey & 12.3 & 9.9 & 14.3 & 3.3 & 3.9 & 3.6 \\
\hline 60 & Nazilli M-503 & Turkey & 14.9 & 10.5 & 12.5 & 4.1 & 3.9 & 4.0 \\
\hline 61 & NGF-63 & Turkey & 12.3 & 9.9 & 11.1 & 3.8 & 4.0 & 3.9 \\
\hline 62 & Barut 2005 & Turkey & 11.9 & 10.0 & 11.0 & 5.2 & 4.1 & 4.6 \\
\hline 63 & Menderes 2005 & Turkey & 8.3 & 8.0 & 8.3 & 4.9 & 4.1 & 4.5 \\
\hline 64 & NAPA 122 & Turkey & 10.1 & 10.3 & 10.3 & 4.9 & 3.8 & 4.3 \\
\hline 65 & NP Ege 2009 & Turkey & 12.3 & 10.4 & 11.4 & 6.3 & 4.6 & 5.4 \\
\hline 66 & NP Özbek 100 & Turkey & 13.3 & 8.7 & 10.7 & 4.3 & 5.3 & 4.8 \\
\hline 67 & SamarkantUzbek & Uzbekistan & 11.3 & 9.2 & 10.2 & 4.6 & 3.6 & 4.1 \\
\hline 68 & Taşkent 1 & Uzbekistan & 9.9 & 7.7 & 8.7 & 4.6 & 4.0 & 4.3 \\
\hline 69 & Taşkent-6 & Uzbekistan & 12.4 & 9.5 & 10.9 & 3.7 & 3.3 & 3.5 \\
\hline 70 & Taşkent Uzbek & Uzbekistan & 10.1 & 7.9 & 8.9 & 5.2 & 4.2 & 4.7 \\
\hline 71 & $152 \mathrm{~F}$ & Uzbekistan & 8.2 & 8.0 & 8.2 & 5.3 & 4.4 & 4.9 \\
\hline 72 & Aleppo-1 & Syria & 8.2 & 7.7 & 8.0 & 5.9 & 4.6 & 5.3 \\
\hline 73 & S-9 & Syria & 7.4 & 8.1 & 7.8 & 5.4 & 4.3 & 4.8 \\
\hline 74 & Delcerro & USA & 11.4 & 6.1 & 8.4 & 3.9 & 5.0 & 4.3 \\
\hline 75 & Delcerro MS-30 & USA & 10.8 & 7.7 & 9.2 & 4.8 & 4.0 & 4.4 \\
\hline 76 & Sindos 80 & Greece & 11.1 & 9.7 & 10.4 & 4.4 & 3.7 & 4.0 \\
\hline 77 & Zeta 2 & Greece & 9.7 & 8.1 & 8.9 & 5.6 & 4.4 & 5.0 \\
\hline 78 & AZ 31 & Israil & 8.7 & 7.1 & 7.9 & 6.4 & 5.5 & 5.9 \\
\hline 79 & Eva & Greece & 11.6 & 7.3 & 9.2 & 5.4 & 5.1 & 5.2 \\
\hline 80 & GW Teks & USA & 11.5 & 6.3 & 8.5 & 5.0 & 4.9 & 4.9 \\
\hline 81 & NIAB 111 & Pakistan & 11.3 & 8.6 & 9.9 & 4.8 & 4.5 & 4.6 \\
\hline 82 & NIAB 999 & Pakistan & 8.7 & 6.0 & 7.2 & 6.2 & 6.5 & 6.3 \\
\hline 83 & Tamcot 22 & USA & 12.8 & 8.8 & 10.6 & 5.4 & 2.9 & 4.0 \\
\hline 84 & Tamcot Sphinx & USA & 9.8 & 6.0 & 7.6 & 5.4 & 5.0 & 5.2 \\
\hline 85 & SJ- U 86 & USA & 11.5 & 7.6 & 9.4 & 6.2 & 3.9 & 5.0 \\
\hline 86 & Candia & Australia & 10.4 & 6.9 & 8.4 & 4.7 & 4.7 & 4.6 \\
\hline 87 & Celia & Australia & 11.6 & 6.9 & 8.9 & 5.5 & 4.6 & 5.1 \\
\hline 88 & Elsa & Australia & 12.5 & 7.8 & 9.9 & 5.2 & 4.7 & 5.0 \\
\hline 89 & Delta Diomand & Spain & 12.2 & 9.4 & 10.7 & 5.2 & 4.0 & 4.6 \\
\hline 90 & Gloria & Australia & 12.1 & 7.2 & 9.3 & 5.6 & 4.7 & 5.2 \\
\hline 91 & Julia & Australia & 12.1 & 7.3 & 9.4 & 4.4 & 4.4 & 4.4 \\
\hline 92 & Flora & Australia & 10.0 & 6.7 & 8.2 & 5.4 & 4.7 & 5.0 \\
\hline 93 & PG 2018 & Turkey & 9.7 & 7.7 & 8.7 & 5.3 & 4.2 & 4.7 \\
\hline 94 & BA 308 & Turkey & 7.8 & 6.4 & 7.1 & 6.1 & 4.9 & 5.5 \\
\hline 95 & BA 525 & Turkey & 9.6 & 9.4 & 23.9 & 3.9 & 4.5 & 4.1 \\
\hline \multirow[t]{10}{*}{96} & Lider & Turkey & 10.4 & 10.7 & 10.6 & 4.8 & 3.2 & 4.0 \\
\hline & Mean & & 11.0 & 8.0 & 9.6 & 4.8 & 4.7 & 4.7 \\
\hline & \multicolumn{8}{|l|}{ Check Varieties } \\
\hline & Carmen & & 10.5 & 8.6 & 9.4 & 4.5 & 5.0 & 4.7 \\
\hline & Şahin 2000 & & 12.5 & 10.3 & 11.5 & 4.5 & 3.4 & 3.9 \\
\hline & BA 119 & & 10.6 & 8.3 & 9.3 & 4.6 & 4.8 & 4.6 \\
\hline & GSN 12 & & 11.9 & 8.6 & 10.0 & 4.6 & 4.7 & 4.6 \\
\hline & Claudia & & 12.1 & 8.3 & 10.0 & 4.4 & 4.0 & 4.2 \\
\hline & Mean & & 11.2 & 8.8 & $\mathbf{1 0 . 0}$ & 4.5 & 4.4 & 4.4 \\
\hline & $\mathrm{LSD}_{0.05}$ & & 4.6 & 4.2 & 3.2 & 2.4 & 3.9 & 2.7 \\
\hline
\end{tabular}

${ }^{* *}$ FI: Full irrigation, DI: Deficit irrigation, GMP: Geometric mean productivity. 
Table 3. Correlation coefficients among full irrigation, deficit irrigation, geometric mean productivity, and drought susceptibility index for seed cotton yield, lint percentage, boll number, and boll weight for 96 cotton genotypes.

\begin{tabular}{|c|c|c|c|c|c|c|c|c|c|c|c|c|c|}
\hline & & \multicolumn{3}{|c|}{$\begin{array}{l}\text { Seed cotton yield } \\
\left(\mathrm{kg} \mathrm{ha}^{-1}\right)\end{array}$} & \multicolumn{3}{|c|}{$\begin{array}{c}\text { Lint percentage } \\
(\%)\end{array}$} & \multicolumn{3}{|c|}{$\begin{array}{l}\text { Boll number } \\
\left.\text { (boll plant }^{-1}\right)\end{array}$} & \multicolumn{3}{|c|}{$\begin{array}{l}\text { Boll weight } \\
\text { (g) }\end{array}$} \\
\hline & & DI & GMP & DSI & FI & DI & GMP & FI & DI & GMP & FI & DI & GMP \\
\hline \multirow[t]{4}{*}{ Seed cotton yield } & FI & $0.42 * *$ & $0.88 * *$ & $0.68 * *$ & 0.09 & 0.10 & 0.01 & 0.14 & 0.12 & $0.20^{*}$ & $0.40 * *$ & 0.05 & $0.30 * *$ \\
\hline & DI & & $0.81 * *$ & $-0.37 * *$ & 0.11 & 0.15 & 0.14 & 0.11 & $0.22 *$ & $0.19 *$ & 0.16 & $0.42 * *$ & $0.36 * *$ \\
\hline & GMP & & & $0.25^{* *}$ & 0.12 & 0.15 & 0.14 & 0.15 & $0.20 *$ & $0.23^{*}$ & $0.35 * *$ & $0.26^{* *}$ & $0.39 * *$ \\
\hline & DSI & & & & 0.02 & -0.01 & 0.01 & 0.05 & -0.04 & 0.05 & $0.29 * *$ & $-0.27 * *$ & 0.02 \\
\hline \multirow[t]{3}{*}{ Lint percentage } & FI & & & & & $0.87 * *$ & $0.96 * *$ & 0.11 & -0.01 & 0.09 & -0.14 & 0.06 & -0.05 \\
\hline & DI & & & & & & $0.97 * *$ & 0.07 & 0.01 & 0.06 & -0.09 & 0.09 & 0.01 \\
\hline & GMP & & & & & & & 0.09 & 0.01 & 0.08 & -0.11 & 0.08 & -0.02 \\
\hline \multirow[t]{3}{*}{ Boll number } & FI & & & & & & & & $0.20 *$ & $0.91 * *$ & $-0.41 * *$ & -0.04 & $-0.31 * *$ \\
\hline & DI & & & & & & & & & $0.58 * *$ & $-0.25 * *$ & $-0.59 * *$ & $-0.52 * *$ \\
\hline & GMP & & & & & & & & & & $-0.48 * *$ & $-0.28 * *$ & $-0.49 * *$ \\
\hline \multirow[t]{2}{*}{ Boll weight } & FI & & & & & & & & & & & $0.27 * *$ & $0.81 * *$ \\
\hline & DI & & & & & & & & & & & & $0.78 * *$ \\
\hline
\end{tabular}

* Indicates significant at $\mathrm{P}=0.05$

*** Indicates significant at $\mathrm{P}=0.01$

The result of a biplot analysis is shown in Figure 1 which is divided into four quadrants. In biplot, quadrant I demonstrate 23 genotypes which are not only water stress tolerant but also give maximum seed cotton yield (No: 10, $21,31,40,42,47,50,51,53,54,57,59,61,64,77,79$, $81,82,84,86,89,91$, and 93). Quadrant II, includes 27 genotypes which are fairly tolerant to water stress but produced lower production (No: 2, 3, 4, 7, 11, 12, 13, 14, $15,16,27,30,33,36,37,38,44,46,49,52,56,69,71$,
74, 75, 76, and 96). Quadrant III, represents 26 genotypes which are highly susceptible to water stress but produced high yield in full irrigation (No: 8, 17, 20, 23, 24, 26, 28, $29,34,39,43,45,55,58,60,62,65,66,72,78,80,85,88$, 90, 94, and 95). Quadrant IV corresponds to susceptible 20 genotypes with lower yields (No: 1, 5, 6, 9, 18, 19, 22, $25,32,35,41,48,63,67,68,70,73,83,87$, and 92).

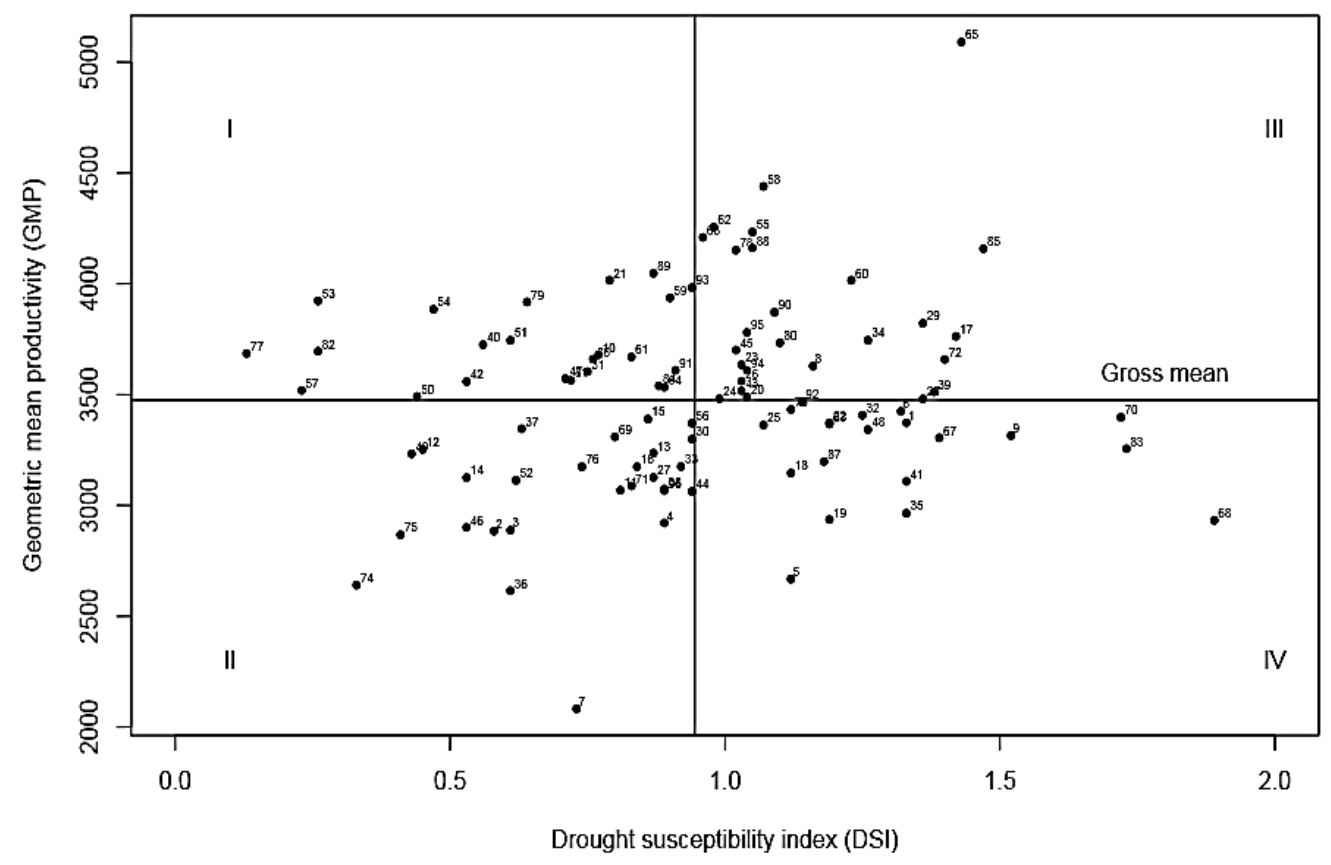

Figure 1. Biplot between geometric mean productivity (GMP) and drought susceptibility index (DSI) values for cotton genotypes.

Drought is the most significant environmental stress in global agriculture. Therefore, the major plant breeding goal is to develop germplasm with improved yield potential under drought conditions (Cattivelli et al., 2008). Existance of useful genetic variation for specific traits releted to drought tolerance in parental germplasm is crucial for successful improvement of crop cultivars (Teran and Singh, 2002). Thus, during recent years the cotton breeders throughout the world have started to develop cotton materials bringing genetic modification in the elite cultivars as parents of new populations, and also utilizing new germplasm in their breeding programme (Iqbal et al., 1997, Basal et al., 2005; Iqbal et al., 2005; Ullah et al., 2006). The difficulty in identification of a physiological parameter as a reliable indicator of yield in drought conditions has suggested that yield performance 
over a range of environments should be used as the main indicator for drought tolerance in rice (Voltas et al., 2005), barley (Rizza et al., 2004), maize (Tollenaar and Lee, 2002), sugar beets (Ober et al., 2004), and cotton (Ullah et al., 2006). In this study, overall cotton genotypes mean performance for yield and yield components in stress conditions was low as compared to non-stress conditions, nevertheless some genotypes exhibited less mean difference in both irrigation regimes, thus showing genotypic variation for drought tolerance.

Significant and positive correlations between seed cotton yield under DI and boll number and boll weight under DI shows that these two yield components could be good indicators to determine water stress tolerant cotton genotypes. Positive correlation between seed cotton yield in DI and FI shows that high yielding in the DI was also high yielding in FI environment. These results agree with those reported by Ramirez Vallejo and Kelly (1998) and Teran and Singh (2002) in common bean, Abdi et al. (2013) in sunflower, Khokhar et al. (2012) in barley. However, there have been contradictory reports in the literature, Gholipouri et al. (2009) and Anwar et al. (2011) reported that, the yield under irrigated conditions has a very weak association with stress conditions, therefore, indirect selection for stresses environment based on the performance of irrigated conditions would not be effective in wheat. Rosielle and Hamblin (1981) predicted that high yielding genotypes in drought stress were likely to be low yielding in well-watered invironments. Negative association between seed cotton yield in DI and DSI would be expected because a higher yield in DI should result in lower percentage reduction and DSI values. Significant negative association of DSI with cotton yield in DI suggested DSI as a useful predictor of drought tolerance in cotton. These findings also support those of Rashid et al. (1999), Moinuddin et al. (2005), and Ullah et al. (2006) who reported that DSI might provide a more effective mean to assess drought tolerance in crops. However, positive association between GMP and DSI suggested that cotton genotypes having high GMP for yield also may result in high reduction in yield (DSI value higher than (1) under DI environment. On the other hand, GMP was positively and significantly correlated with seed cotton yield in DI. Regression analysis also showed that GMP had positive and DSI had negative effect on seed cotton yield under water stressed condition. This result indicates that cotton genotypes with high GMP and low DSI would be selected as drought tolerant genotypes. Our results corroborate those of Ullah et al. (2006), who reported that selection for combination of DSI and GMP indices might be more useful in improving drought tolerance in cotton instead of using a single yield basis criterion since each index is a potential indicator of different biological responses to drought.

\section{CONCLUSION}

The present study was aimed to examine drought tolerance of a set of Upland cotton genotypes under FI and DI regimes. Seed cotton yield and its components of 96 cotton genotypes were markedly affected DI regime.
Water stress caused a significant variation in yield and ranged from $1,791 \mathrm{~kg} \mathrm{~h}^{-1}$ to $3,993 \mathrm{~kg} \mathrm{~h}^{-1}$. The results demonstrated that genetic variability for water stress tolerance existed in the material examined. Significant and positive correlation between seed cotton yields in DI and FI shows that indirect selection based on the performance of irrigated conditions would be used for genetic improvement in cotton under stressful environment. Based on correlation and regression analysis, GMP and DSI could be used as reliable screening criteria for drought resistance. It is concluded from the present study that based on biplot analysis, 23 cotton genotypes were identified suitable for stress conditions, and GC 555, Nieves, DAK-66/3, MS-30/1, Nazilli M-503, Zeta 2, Eva, NIAB 999, and Delta Diomand were found highly water stress tolerant due to the high GMP and low DSI values. These genotypes could be used as genetic resources for improving seed cotton yield productivity under drought conditions.

\section{ACKNOWLEDGEMENTS}

This project was supprted by the Scientific and Technological Research Council of Turkey -TÜBİTAK, Project No: 3110087.

\section{LITERATURE CITED}

Abdi, N., R. Darvishzadeh and H.H. Maleki. 2013. Effective selection criteria for screening drought tolerant recombinant inbred lines of sunflower. Genetika. 45:153-166.

Al-Hamdani, S.H. and T.W. Barger. 2003. Influence of water stress on selected physiological responses of three sorghum genotypes. Italy Journal Agronomy. 7: 15-22.

Anwar, J., G.M. Subhan, M. Hussain, J. Ahmad, M. Hussain and M. Munir. 2011. Drought tolerance indices and their correlation with yield in exotic wheat genotypes. Pak J Bot. 43:1527-1530.

Basal, H, N. Dagdelen, A. Unay and E. Yilmaz. 2009. Effects of defi cit drip irrigation ratios on cotton (Gossypium hirsutum L.) yield and fiber quality. J. Agron. Crop Sci. 195: 19-29.

Basal, H. C.W. Smith, P.M. Thaxton and J.K. Hemphill. 2005. Seedling drought tolerance in upland cotton. Crop Sci. 45: 766-771.

Bednarz, C.W., J.Hook, R. Yager, S. Cromer, D. Cook, and I. Griner. 2002. Cotton crop water use and irrigation scheduling, p. 61-64, In A. S. Culpepper, ed. 2002 Georgia Cotton Research-Extension Report.

Cattivelli, L., F. Rizza, F.W. Badeck, E. Mazzucotelli, A.M. Mastrangelo, E. Francia, C. Marè, A. Tondelli and A.M. Stanca. 2008. Drought tolerance improvement in crop plants: An integrated view from breeding to genomics. Field Crops Research. 105: 1-14.

Fernandez, G.C.J. 1992. Effective selection criteria for assessing plant stress tolerance. p. 257-270. In C.G. Kuo (ed.) Adaptation of food crops to temperature and water stress. p. 531. In Proc. Int. Symp., Taipei, Taiwan. Aug13-18. Pp: 93410.

Fischer, R.A. and R. Maurer. 1978. Drought resistance in spring wheat cultivars. I. Grain yield responses. Aust. J. Agric. Res. 29: 897-912.

Gholipouri, A. M. Sedghi, R.S. Sharifi and N.M. Nazari. 2009. Evaluation of drought tolerance indices and their relationship with grain yield in wheat cultivars. Recent Res. Science Technolgy. 1: 195-198. 
Guinn, G. and J.R. Mauney. 1984. Moisture effects on cotton. I. Effects of moisture status on flowering. Agronomy Journal. 76: $90-94$

Iqbal, M.J., N. Aziz, N.A. Saeed, Y. Zafer and K.A. Malik. 1997. Genetic Diversity evaluation of some elite cotton varieties by RAPD analysis. Theor. Appl. Genetic. 94: 139144.

Iqbal, M. N. Nisar, R.S.A. Khan and K. Hayat. 2005. Contribution of mepiquat chloride in drought tolerance in cotton seedlings. Asian J. PI. Science. 4: 530-532.

Khokhar, M.L., J.A. Teixeira da Silva and H. Spiertz. 2012. Evaluation of barley genotypes for yielding ability and drought tolerance under irrigated and water-stressed conditions. American-Eurasian J Agric Environ Sci. 12: $287-$ 292.

Lacape, M.J., J. Wery and D.J.M. Annerosa. 1998. Relationship between plant and soil water status in five field-growing cotton (Gossypium hirsutum L.) cultivars. Field Crops Res. 57:29-48.

Loka, D.A., D.M. Oosterhuis and G.L. Ritchie. 2011. Stress Physiology in Cotton: Water-deficit stress in cotton. The Cotton Foundation, Cordova, Tennessee (USA), pp: 37-72.

Massaci, A. S.M. Nabiev, L. Petrosanti, S.K. Nematov, T.N Chernikova, K. Thor and J. Leipner. 2008. Response of the photosynthetic apparatus of cotton (Gossypium hirsutum L.) to the onset of drought stress under field conditions studied by gas-exchange analysis and chlorophyll fluorescence imaging. Plant Physiol. Biochem. 46: 189-195.

Mert, M. 2005. Irrigation of cotton cultivars improves seed cotton yield, yield components and fibre properties in the Hatay region, Turkey. Acta Agronomy Scand. 55: 44-50.

Moinuddin, R.A. Fischer, K.D. Sayre and M.P. Reynolds. 2005. Osmotic Adjustment in wheat in relation to grain yield under water deficit environments. Agronomy Journal 97: 10621071.

Ober, E.S., C.J.A. Clark, M. Le Bloa, A. Royal, K.W. Jaggard and J.D. Pidgeon. 2004. Assessing the genetic resources to improve drought tolerance in Sugar beet: Agronomic traits of diverse genotypes under droughted and irrigated conditions. Field Crops Research. 90: 213-234.

Pettigrew, W.T. 2004. Moisture deficit effects on cotton lint yield, yield components, and boll distribution. Agronomy Journal. 96: 377-383.

Passioura, J.B. 2006. The perils of pot experiments. Functional Plant Biology. 33:1075-1079.
Pettigrew, W.T. and W.R. Meredith. 1994. Leaf gas exchange parameters vary among cotton genotypes. Crop Sci. 34:700705.

Quisenberry, J.E., W.R. Jordan, B.A. Roark and D.W. Fryrear. 1981. Exotic cottons as genetic sources for drought resistance. Crop Sci. 21: 889-895.

Ramirez-Vallejo, P. and J.D. Kelly. 1998. Traits related to drought resistance in common bean. Euphytica. 99: 127-136.

Rashid, A., J.C. Stark, A. Tanveer and T. Mustafa. 1999. Use of canopy temperature measurements as a screening tool for drought tolerance in spring wheat. J. Agron. Crop Science. 182: 231-237.

Ritchie, G.L., C.W. Bednarz, P.H. Jost and S.M. Brown. 2004. Cotton Growth and Development. Bulletin 1252. Cooperative Extension Service and the University of Georgia College of Agricultural and Environmental Sciences, Athens, GA, USA.

Rizza, F., F.W. Badeck, L. Cattivelli, L. Destri, O. Di Fonzo and N. Stanca. 2004. Use of a water stress index to identify barley genotypes adapted to rainfed and irrigated conditions. Crop Sci. 44: 2127-2137.

Rosielle, A.A. and J. Hamblin. 1981. Theoretical aspects of selection for yield in stress and non- stress environment. Crop Sci. 21: 943-946.

Sinclair, T.R. 2005. Theoretical analysis of soil and plant traits influencing daily plant water flux on drying soils. Agronomy Journal. 97: 1148-1152.

Terán, H. and S.P. Singh. 2002. Comparison of sources and lines selected for drought resistance in common bean. Crop Sci. 42: 64-70.

Tollenaar, M. and E.A. Lee. 2002. Yield stability and stress tolerance in maize. Field Crops Research. 75: 161-169.

Turner, N.C. 1997. Further progress in crop water relations. Advance Agronnomy 58: 293-338.

Ullah, I. M. Rahman and Y. Zafar. 2006. Genotypic varıation for drought tolerance in cotton (Gossypium hirsutum L.) Seed cotton yield responses. Pak. J. Bot. 38: 1679-1687.

Voltas, J., H. Lopez-Corcoles and G. Borras. 2005. Use of biplot analysis and factorial regression for the investigation of superior genotypes in multi environment trials. Eur. Journal Agronomy. 22: 309-324.

Wang, C. A. Isoda and P. Wang. 2004. Growth and yield performance of some cotton cultivars in Xinjiang, China, an arid area with short growing period. J. Agronomy Crop Science. 190: 177-183. 